\title{
Corticolimbic fast-tracking: enhanced multimodal integration in functional neurological disorder
}

\author{
Ibai Diez, ${ }^{1,2,3,4}$ Laura Ortiz-Terán, ${ }^{1,4}$ Benjamin Williams, ${ }^{1}$ Rozita Jalilianhasanpour, ${ }^{\oplus 1}$ \\ Juan Pablo Ospina, ${ }^{1}$ Bradford C Dickerson, ${ }^{1}$ Matcheri S Keshavan, ${ }^{5}$ \\ W Curt LaFrance $\mathrm{Jr}_{1}{ }^{6}$ Jorge Sepulcre, ${ }^{4}$ David L Perez ${ }^{0,7}$
}

\begin{abstract}
- Additional material is published online only. To view please visit the journal online (http://dx.doi.org/10.1136 jnnp-2018-319657).
\end{abstract}

For numbered affiliations see end of article.

Correspondence to Dr David L Perez, Neurology and Psychiatry, Massachusetts General Hospital, Charlestown MA 02129,USA; dlperez@ partners.org

JS and DLP contributed equally.

Received 15 September 2018 Revised 18 December 2018 Accepted 9 February 2019 Published Online First 8 March 2019

\section{SLinked}

- http://dx.doi.org/10.1136/ jnnp-2019-320597

Check for updates

(C) Author(s) (or their employer(s)) 2019. No commercial re-use. See rights and permissions. Published by BMJ.

To cite: Diez I, Ortiz-Terán L, Williams B, et al. J Neurol Neurosurg Psychiatry

2019:90:929-938.

\section{ABSTRACT}

Objective Some individuals with functional neurological disorder (FND) exhibit motor and affective disturbances, along with limbic hyper-reactivity and enhanced motor-limbic connectivity. Given that the multimodal integration network (insula, dorsal cingulate, temporoparietal junction (TPJ)) is implicated in convergent sensorimotor, affective and interoceptive processing, we hypothesised that patients with FND would exhibit altered motor and amygdalar resting-state propagation to this network. Patient-reported symptom severity and clinical outcome were also hypothesised to map onto multimodal integration areas.

Methods Between-group differences in primary motor and amygdalar nuclei (laterobasal, centromedial) were examined using graph-theory stepwise functional connectivity (SFC) in 30 patients with motor FND compared with 30 healthy controls. Within-group analyses correlated functional propagation profiles with symptom severity and prospectively collected 6-month outcomes as measured by the Screening for Somatoform Symptoms Conversion Disorder subscale and Patient Health Questionnaire-15 composite score. Findings were clusterwise corrected for multiple comparisons.

Results Compared with controls, patients with FND exhibited increased SFC from motor regions to the bilateral posterior insula, TPJ, middle cingulate cortex and putamen. From the right laterobasal amygdala, the FND cohort showed enhanced connectivity to the left anterior insula, periaqueductal grey and hypothalamus among other areas. In within-group analyses, symptom severity correlated with enhanced SFC from the left anterior insula to the right anterior insula and TPJ; increased SFC from the left centromedial amygdala to the right anterior insula correlated with clinical improvement. Within-group associations held controlling for depression, anxiety and antidepressant use.

Conclusions These neuroimaging findings suggest potential candidate neurocircuit pathways in the pathophysiology of FND.

\section{INTRODUCTION}

Despite its important role in the development of modern-day neurology and psychiatry, functional neurological disorder (FND, also known as conversion disorder) has been largely neglected by both fields for decades. ${ }^{12}$ However, the reframing of FND as a 'rule-in' diagnosis based on neurological signs and semiological features has catalysed renewed interest. ${ }^{34}$ Improved diagnostic specificity offers the opportunity to elucidate the pathophysiology of this enigmatic condition, which in turn could challenge mind-body dualism, reduce patient stigma and aid the development of neurobiologically informed treatments.

Several neuroimaging studies in FND cohorts have identified amygdalar abnormalities including hyper-reactivity to affectively valenced stimuli, ${ }^{5-8}$ impaired habituation, ${ }^{7}$ sensitised emotional processing $^{5}$ and heightened coupling to motor preparation areas including the supplementary motor area (SMA). ${ }^{67910}$ For example, patients with FND performing an affectively valenced face viewing task demonstrated increased amygdalar activity and delayed habituation compared with controls. ${ }^{7}$ These findings were replicated in another FND study that also showed increased dorsal anterior cingulate cortex (ACC), periaqueductal grey (PAG) and SMA activations during emotional processing in patients compared with controls. ${ }^{5}$ Increased task-based ${ }^{67}$ and resting-state ${ }^{811}$ amygdalar coupling to motor and cognitive control regions has also been described. In addition, cingulo-insular resting-state connectivity to sensorimotor areas is increased in some FND populations, ${ }^{12}$ and these connectivity relationships correlate with symptom severity. ${ }^{13}$ While important unanswered questions remain, such as the role of specific amygdalar nuclei, these findings support salience network (amygdala, cingulo-insular, PAG) involvement in the pathophysiology of FND.

Neuroimaging findings related to altered motor preparation, execution, inhibitory control and conceptualisation have also been reported in patients with FND. ${ }^{14-16}$ For example, patients with functional paralysis attempting to move an affected limb exhibited an expanded network of insular, ventrolateral prefrontal cortex, basal ganglia and lingual gyrus activations. ${ }^{17}$ During the execution of internally generated movements in individuals with functional movement disorders, similar anterior insular and amygdalar hyperactivations were observed. ${ }^{18}$ Amplified startle responses have also been described in individuals with FND. ${ }^{19}$ While heightened limbic-paralimbic influence on behaviour has been proposed as an important aspect of the pathophysiology of $\mathrm{FND},{ }^{7}$ the specific pathways through which motor and affective neural systems interact in FND remain poorly understood.

Stepwise functional connectivity (SFC) is a novel graph-theory resting-state functional connectivity 
approach developed by Sepulcre and colleagues that characterises the propagation and convergence of functional connectivity across brain networks. ${ }^{20-22}$ Conventional resting-state methods predominantly characterise the segregation of discrete largescale networks; SFC is specifically designed to capture the segregation of brain networks and the integration between them, as a proxy of the information flow across neuronal systems. Using this method, sensorimotor systems in healthy subjects have been observed to propagate over a series of functional connectivity relay stations (link-steps) to a core set of multimodal integration areas. ${ }^{2021}$ The multimodal integration network includes the anterior insula, dorsal $\mathrm{ACC} /$ middle cingulate cortex (MCC), ventral premotor cortex and temporoparietal junction (TPJ). Anterior portions of the multimodal integration network overlap with the salience network, while posterior areas of this network (eg, TPJ) are implicated in motor intention awareness and action authorship deficits in FND. ${ }^{23-25}$ Notably, SFC has characterised mechanistic insights in neurologic ${ }^{26}$ and neuropsychiatric populations. ${ }^{27}$

In this resting-state neuroimaging study, we examined the functional propagation of primary motor (hand, foot, tongue) and amygdalar (laterobasal, centromedial nuclei) regions in 30 patients with motor FND compared with 30 healthy controls. A SFC interconnector analysis also explored common points of altered functional connectivity between motor and amygdalar areas in patients with FND compared with controls. Complementary within-group approaches investigated correlations between SFC profiles, patient-reported symptom severity and 6-month clinical outcomes. We have previously characterised salience network structural alterations in this cohort, ${ }^{28} 29$ and have theorised that cingulo-insular areas contribute to impaired integration of sensorimotor, affective and viscerosomatic information in FND populations. ${ }^{30}$ Thus, we hypothesised that individuals with motor FND would exhibit altered motor and amygdalar link-step connectivity to the multimodal integration network. We also hypothesised that FND symptom severity and prospectively collected 6-month clinical outcome would correlate with multimodal integration network functional connectivity profiles.

\section{METHODS}

Adapted from refs 20-22.

\section{Participants and questionnaires}

Thirty subjects with motor FND ( 24 women, 6 men; mean age $=40.1 \pm 12.9$; average illness duration $=3.0 \pm 3.8$ years; 24 right handed, 6 ambidextrous or left handed) were recruited from the Massachusetts General Hospital (MGH) FND Clinic following a 'rule-in' FND diagnosis in accord with the Diagnostic and Statistical Manual of Mental Disorders Fifth Edition criteria. ${ }^{3}$ An additional five patients were enrolled but excluded following imaging preprocessing (see online supplementary methods). Given the overlap across the motor FND spectrum, ${ }^{30}$ we used a transdiagnostic approach that included those with clinically established functional movement disorders $(n=16),{ }^{31}$ functional weakness $(n=12)^{32}$ and documented $(n=12)$ or clinically established $(\mathrm{n}=1)$ psychogenic non-epileptic seizures (PNES). ${ }^{33}$ Ten of the 30 subjects had mixed motor FND. Exclusion criteria included major neurological comorbidities with MRI abnormalities, epilepsy, poorly controlled medical problems with known central nervous system consequences, active substance dependence, a history of mania or psychosis and/or active suicidality. Comorbid psychiatric diagnoses as assessed using the Structured Clinical Interview for DSM-IV-TR (SCID-I) were present in 27 of 30 participants. Fourteen patients were on selective serotonin reuptake inhibitors (SSRI) and/or serotonin-norepinephrine reuptake inhibitors (SNRI). See online supplementary table 1 for clinical information. Thirty healthy subjects ( 22 women, 8 men; mean age $=40.0 \pm 12.6 ; 25$ right handed, 5 ambidextrous or left handed) were recruited through local advertisements, and all screened negative for SCID-I major psychiatric comorbidities (one control met criteria for past depression not otherwise specified). Five additional controls were enrolled but excluded due to lifetime major psychiatric comorbidities $(n=4)$ or excess head motion $(\mathrm{n}=1)$.

Subjects completed the Screening for Somatoform Symptoms-7 Conversion Disorder subscale (SOMS:CD) ${ }^{34}$ and the Patient Health Questionnaire-15 (PHQ15) ${ }^{35}$ as patient-reported symptom severity measures within a detailed psychometric battery at enrolment. The SOMS:CD is a 14-item measure of sensorimotor FND symptoms within the past 7 days, with each item scored on a 5-point scale. The PHQ15 is a 15 -item measure of somatic complaints within the past 4 weeks, with each item scored on a 3-point scale. These scales were also prospectively completed at 6 month follow-up by 22 of 30 patients (eight were lost to follow-up; interval follow-up $=6.1 \pm 1.1$ months). To reduce multiple comparisons, we constructed a SOMS:CD-PHQ15 composite by averaging the z-scores of the two variables. All subjects also completed the Beck Depression Inventory-II (BDI-II) and the Spielberger State-Trait Anxiety Inventory (STAI).

Prior to enrolment, patients were diagnosed with FND and the term 'Functional Neurological Disorder' was communicated along with the pertinent motor subtype. ${ }^{36}$ FND was presented as common, real and treatable. Patients were introduced to the www.neurosymptoms.org website and given printed educational materials. Treatments were individualised, emphasising cognitive-behavioural therapy (CBT) and physiotherapy (PT). Fifteen individuals were in psychotherapy at baseline and eight were newly referred (including one who remained in supportive psychotherapy while also starting CBT). In CBT, patients were encouraged to explore the relationships between functional symptoms, thoughts, behaviours, emotions and psychosocial factors. ${ }^{37}$ Nine were in PT at baseline, and five were newly referred. Physiotherapists were recommended to use the Nielsen et al recommendations. ${ }^{38}$ Patients did not exclusively receive care at MGH, which limited compliance information.

\section{MRI data acquisition and preprocessing}

See online supplementary methods 7 for data acquisition and preprocessing procedures including scrubbing head motion correction.

\section{SFC analyses}

SFC methods delineated the functional propagation of specific regions of interest (ROI) across distinct link-steps. ${ }^{20-22}$ First, individual connectivity matrices were computed. As shown in figure 1 , the Pearson correlation coefficients between the time series of all pairs of cortical-subcortical grey matter voxels were computed. Then, a Fisher transformation was applied to the resulting correlation matrix. After removing negative values due to their controversial interpretation, a false discovery rate correction of q-level $=0.0001$ was applied to obtain only the most significant connections. Thus, a high-resolution $5142 \times 5142$ connectivity matrix was obtained for each subject.

Validated bilateral Montreal Neurological Institute voxelwise hand $( \pm 41,-20,62)$, foot $( \pm 6,-26,76)$ and tongue $( \pm 55,-4$, 


\section{(A) Methods Pipeline}

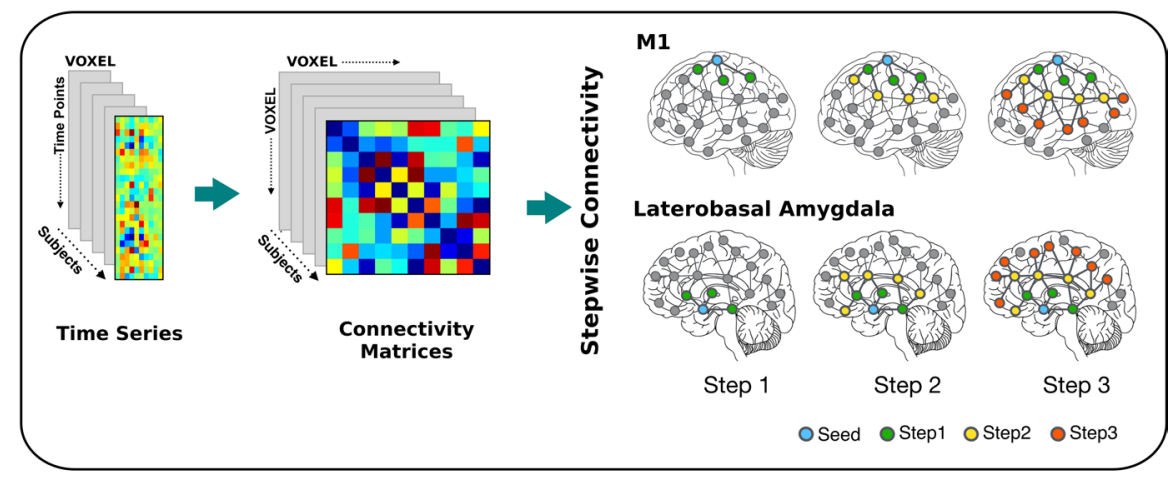

(B) Left M1 Hand (HCs) Step 1
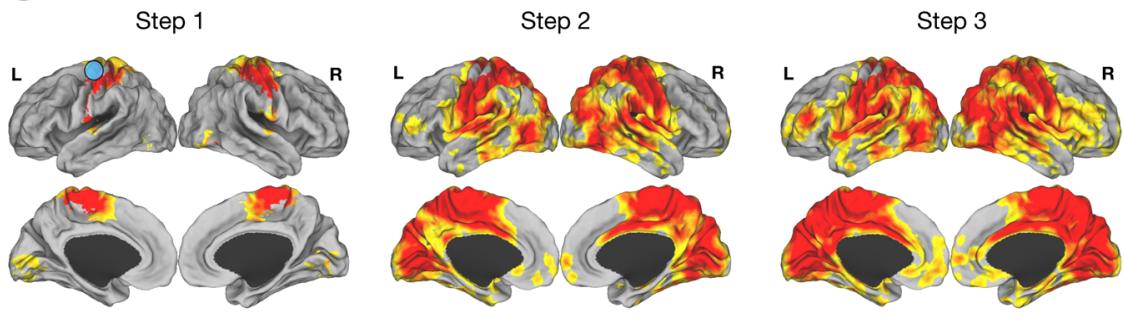

(C) Right Laterobasal Amygdala (HCs)

Step 1

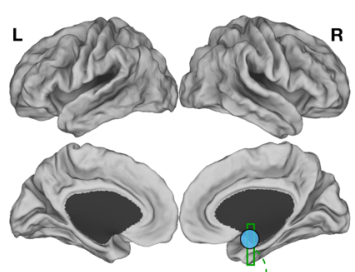

A
Step 2

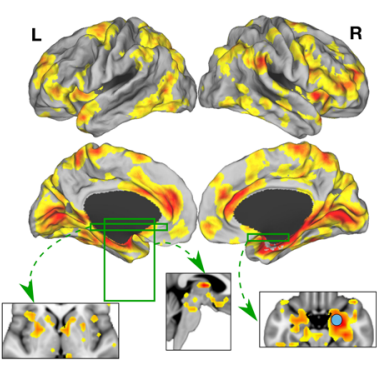

Step 3

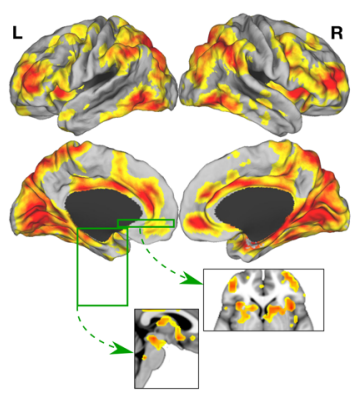

1 sample
T-stat

Figure 1 Stepwise functional connectivity pipeline used to characterise differences in the integration of motor and amygdalar information between patients with functional neurological disorder (FND) and healthy controls (HC). (A) The blood-oxygen-level-dependent time series preprocessed signal was used to create a voxel by voxel connectivity matrix at the individual subject level. Using motor and amygdalar regions of interest (ROI), we computed the step-link connectivity for steps 1, 2 and 3. Green nodes represent the regions reached by the first link-step from the ROI (represented in blue); yellow and red nodes represent second and third link-steps from the ROI. As an illustrative example, panel (B) shows the functional propagation for 30 healthy subjects from the left M1-hand (one-sample t-test threshold at $p=0.01$ corrected for multiple comparisons; reproduced from Sepulcre [20]). Panel (C) shows the functional propagation of the right laterobasal amygdala ROI in healthy subjects; one-sample t-test threshold at $\mathrm{p}=0.01$ corrected for multiple comparisons. The first link-step shows connectivity to adjacent bilateral amygdalar regions. In the second link-step, connectivity is observed to cingulo-insular salience network regions among other areas; in the third link-step, propagation reaches the periaqueductal grey and hypothalamus. See online supplementary figure 1 for delineation of link-step connectivity profiles from other amygdalar nuclei in healthy subjects.

26) primary motor cortex ROIs were chosen. ${ }^{39}$ In addition, laterobasal and centromedial amygdalar ROIs were extracted from the probabilistic cytoarchitectonic mapping of the human amygdala in the Statistical Parametric Mapping (SPM) anatomy toolbox consistent with previously validated approaches ${ }^{40}$ (figure 1 and online supplementary figure 1). Only three linksteps are presented given that healthy subjects reach the multimodal integration network by this stage. ${ }^{2021}$

\section{Between-group analyses}

Two-class general linear models examined between-group differences in the stepwise functional propagation of motor and amygdalar areas. All analyses (including within-group analyses described below) controlled for age, gender and handedness (right handed yes/no). Secondary analyses also adjusted betweengroup findings for depression and anxiety scores. There were no group-level differences in mean frame displacement (FND: $0.098 \pm 0.055$; controls: $0.08 \pm 0.043 ; \mathrm{p}=0.15$ ). Between-group SFC findings were corrected for multiple comparisons using Monte Carlo simulation clusterwise correction with 10000 iterations to estimate the probability of false positive clusters with a p value $<0.05$.

\section{Exploratory between-group interconnector analysis}

While SFC delineates functional connectivity pathways from one region to the rest of the brain, it is not well suited to delineate 
interconnecting paths across multiple ROIs. Interconnector analysis, another graph-theory network analysis, ${ }^{21} 41$ restricts the SFC analysis only to those pathways that end in a common target. Following the identification of between-group SFC differences from a priori ROIs (see the Results section), statistically significant motor and amygdalar ROIs were used in a single interconnector analysis to test for common sites of altered functional propagation. For this exploratory general linear model analysis, a right-tailed uncorrected t-test was used to compute group differences between patients and controls. Only clusters larger than $1.080 \mathrm{~mm}^{3}$ with $\mathrm{p}<0.05$ were reported to minimise type I errors.

\section{Within-group correlation with symptom severity}

A single-class general linear model investigated the effect of symptom severity (SOMS:CD-PHQ15 composite). There was no significant relationship between individual subject frame displacements and composite scores (Spearman correlation 0.24; $\mathrm{p}=0.2$ ). The motor and amygdalar ROIs used for between-group analyses were also used here (although no significant results were identified). We also performed a data-driven approach to identify all voxels where link-step propagation correlated with SOMS:CD-PHQ15 scores, and the left anterior insula was among the most robust areas (online supplementary methods, online supplementary figure 2). Given that we previously characterised inverse correlations between left anterior insular volume and FND symptom severity, ${ }^{28}$ the left anterior insula $(-45$, 9, -9) was chosen as an additional ROI. Correction for multiple comparisons employed clusterwise correction using Monte Carlo simulation. Post hoc analyses evaluated if statistically significant within-group findings held adjusting for baseline: (1) mood and anxiety symptoms (BDI-II and STAI total scores); (2) SSRI/SNRI use (yes/no); and (3) motor FND subtypes (PNES, functional movement disorders and functional weakness).

Pilot within-group correlation with 6-month improvement In the 22 of 30 FND subjects with prospectively collected 6-month follow-up data, a pilot analysis evaluated associations between baseline SFC and clinical outcome. The same motor and amygdalar ROIs were also used here to identify functional propagation profiles correlated with 6-month change in SOMS:CD-PHQ15 scores. The percentage change in the SOMS:CD-PHQ15 composite score was used in a single-class general linear model to study relationships between SFC and clinical outcome (scores were inverted so that higher scores reflected greater improvement). Correction for multiple comparisons applied a clusterwise correction using a Monte Carlo simulation, and post hoc analyses adjusted statistically significant findings for baseline: (1) BDI-II and STAI total scores, and (2) SSRI/SNRI use. Given sample size limitations, we did not adjust for motor subtypes.

\section{RESULTS}

\section{Motor findings}

Compared with controls, the first link-step from left-M1 hand in patients with FND showed increased connectivity to the bilateral precentral and postcentral gyri, SMA, MCC and superior parietal lobule (figure 2). In the second link-step from the left-M1 hand, patients with FND also displayed enhanced propagation to the bilateral posterior insula, TPJ and putamen (in addition to the areas described in the first link-step which were also present). In the third link-step from the left-M1 hand, patients with FND compared with controls also showed greater link-step
(A) Left M1 Hand (FND>HC)
Step 1

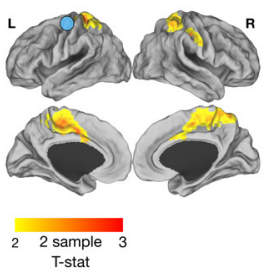

Step 2

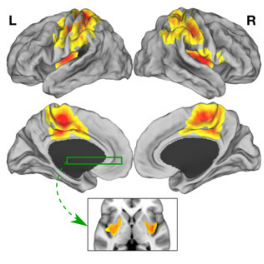

Step 3

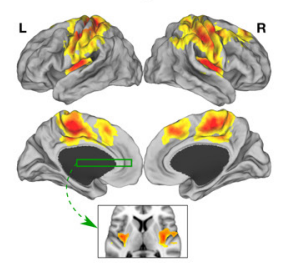

Beft M1 Hand adjusting for anxiety and depression (FND>HC) Step 1

Step 2
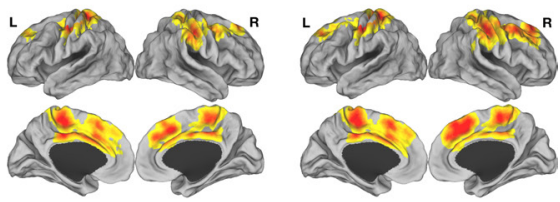

(C) Right M1 Hand (FND>HC)

Step 1
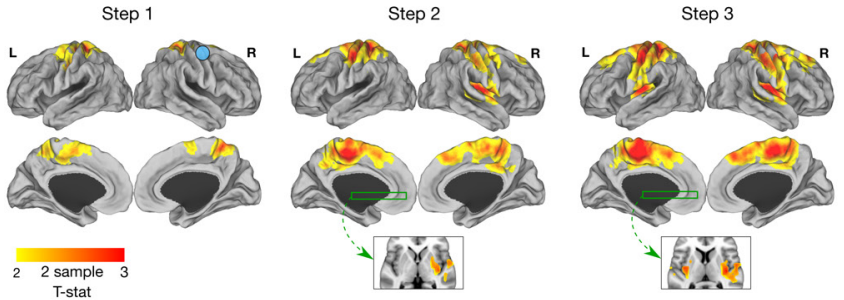

(D) Right M1 Hand adjusting for anxiety and depression (FND>HC) Step 1
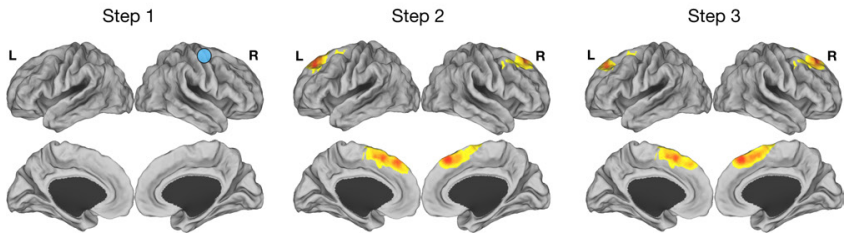

Figure 2 Altered stepwise functional propagation from primary motor areas in patients with functional neurological disorder (FND). Maps display results of general linear models comparing functional propagation in patients with FND versus healthy controls $(\mathrm{HC})$. Only results surviving multiple comparisons are shown adjusting for age, gender and handedness. (A) Patients with FND compared with controls exhibited increased first link-step connectivity from the left M1-hand (right hand projected in left hemisphere) to bilateral precentral and postcentral gyri, supplementary motor area, middle cingulate cortices and superior parietal lobule. In the second link-step from the left M1-hand area, patients with FND compared with controls displayed increased connectivity to the bilateral posterior insula, temporoparietal junction (TPJ) and putamen (in addition to the areas found in the first link-step). In the third link-step, patients with FND showed greater connectivity in bilateral dorsomedial prefrontal areas in addition to the regions observed in earlier steps. (B) Displays left M1-hand functional propagation differences between patients with FND compared with controls adjusting for depression and anxiety scores. (C, D) Results obtained from the right M1-hand in patients with FND compared with controls, which are similar to those observed for the left M1-hand region.

connectivity to bilateral dorsomedial prefrontal areas. Post hoc analyses adjusting for depression and anxiety showed enhanced propagation from the left-M1 hand to the bilateral dorsal ACC/ MCC (in all three link-steps), dorsomedial prefrontal cortex (second and third link-steps) and right TPJ (second and third link-steps) in FND subjects compared with controls.

From the right-M1 hand area, patients with FND showed similar increased SFC profiles across all link-steps to those seen 


\section{(A) Right Laterobasal Amygdala (FND $>\mathrm{HC}$ )}
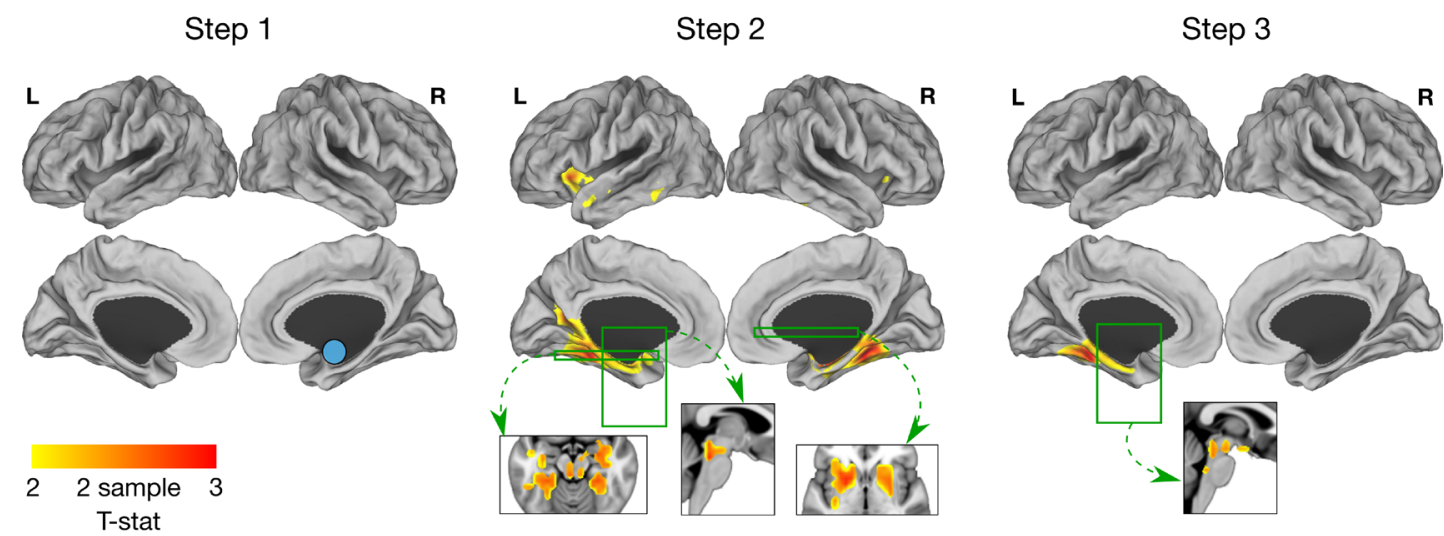

\section{(B) Amygdala-motor interconnector stepwise connectivity analysis}

\section{Interconector analysis}

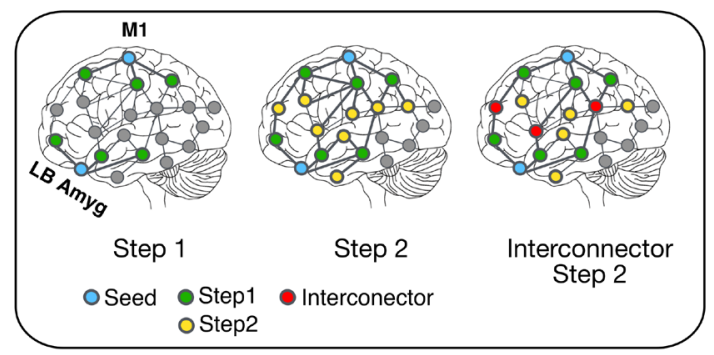

Laterobasal Amygdala - M1 (FND>HC)

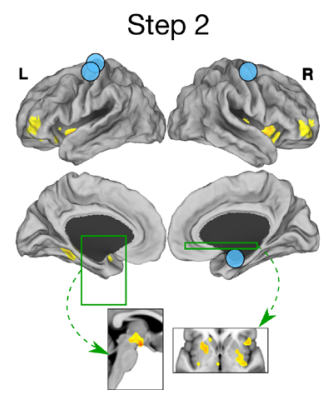

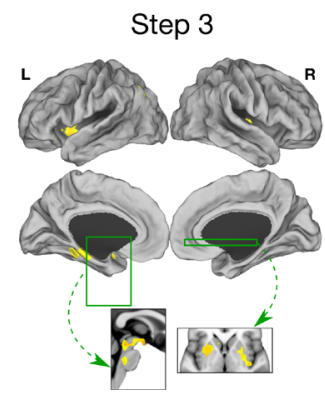

Figure 3 Altered stepwise propagation from the right laterobasal amygdala and an exploratory amygdala-motor interconnector analysis in patients with functional neurological disorder (FND). (A) Patients with FND compared with controls showed greater second link-step connectivity from the right laterobasal amygdala to the left > right anterior insula, bilateral parahippocampal and fusiform gyri, hippocampus, putamen, pallidum and the periaqueductal grey compared with controls. Patients with FND in the third link-step also showed enhanced connectivity to left parahippocampal and fusiform gyri, hypothalamus and the periaqueductal grey compared with controls. These group-level differences in right laterobasal amygdala functional propagation did not remain significant adjusting for depression and anxiety. (B) In the left panel, green nodes represent voxels within one link-step from any of the amygdala or motor regions of interest (ROI, blue nodes). The next link-step from green nodes are displayed in yellow (second link-step). The yellow nodes present in the second link-step from both the amygdala and motor regions are represented in red. The nodes displayed in red represent the interconnector regions where the propagation of information between different ROls converges. In the right panel, uncorrected right-tailed t-test results are displayed. Patients with FND compared with controls showed enhanced second link-step interconnector connectivity to bilateral anterior insula, dorsolateral prefrontal cortices, putamen, left fusiform gyrus and hypothalamus. In the third link-step, higher connectivity was observed in left anterior insula, parahippocampal and fusiform gyri, right posterior insula, bilateral putamen, periaqueductal grey and hypothalamus in patients with FND compared with controls. HC, healthy control; LB, laterobasal.

from the left-M1 hand compared with controls (figure 2). In post hoc analyses adjusting for depression and anxiety, only increased link-step connectivity to the bilateral dorsomedial prefrontal cortex was observed in the second and third steps in patients with FND compared with controls.

Originating from the left-M1 foot, the first link-step did not show any between-group differences. In the second and third link-steps, patients with FND compared with controls demonstrated similar group-level differences as those described for the left and right M1-hand areas (online supplementary figure 3). Group-level differences did not remain significant adjusting for depression and anxiety. No other between-group differences were observed across the six motor ROIs.

\section{Amygdala findings}

From the right laterobasal amygdala, in the second link-step patients with FND displayed enhanced propagation to the left $>$ right anterior insula, bilateral parahippocampal and fusiform gyri, hippocampus, putamen, pallidum and the PAG compared with controls (figure 3). In the third link-step, patients with FND showed increased connectivity to the left parahippocampal and fusiform gyri, PAG and hypothalamus. These between-group findings did not hold adjusting for depression and anxiety scores. There were no other SFC differences across the bilateral amygdala ROIs.

\section{Exploratory interconnector analysis}

When examining common M1-right laterobasal amygdala functional propagation profiles, patients with FND compared with controls showed increased second link-step connectivity to bilateral anterior insula, dorsolateral prefrontal cortex (dlPFC), putamen, left fusiform gyrus and the hypothalamus (figure 3). In the third link-step, patients with FND compared with controls showed greater connectivity to the left anterior insula, parahippocampal and fusiform gyri, right posterior insula, bilateral putamen, PAG and hypothalamus (figure 3). 


\section{(A) Left anterior insular stepwise propagation correlated} with SOMS:CD-PHQ15
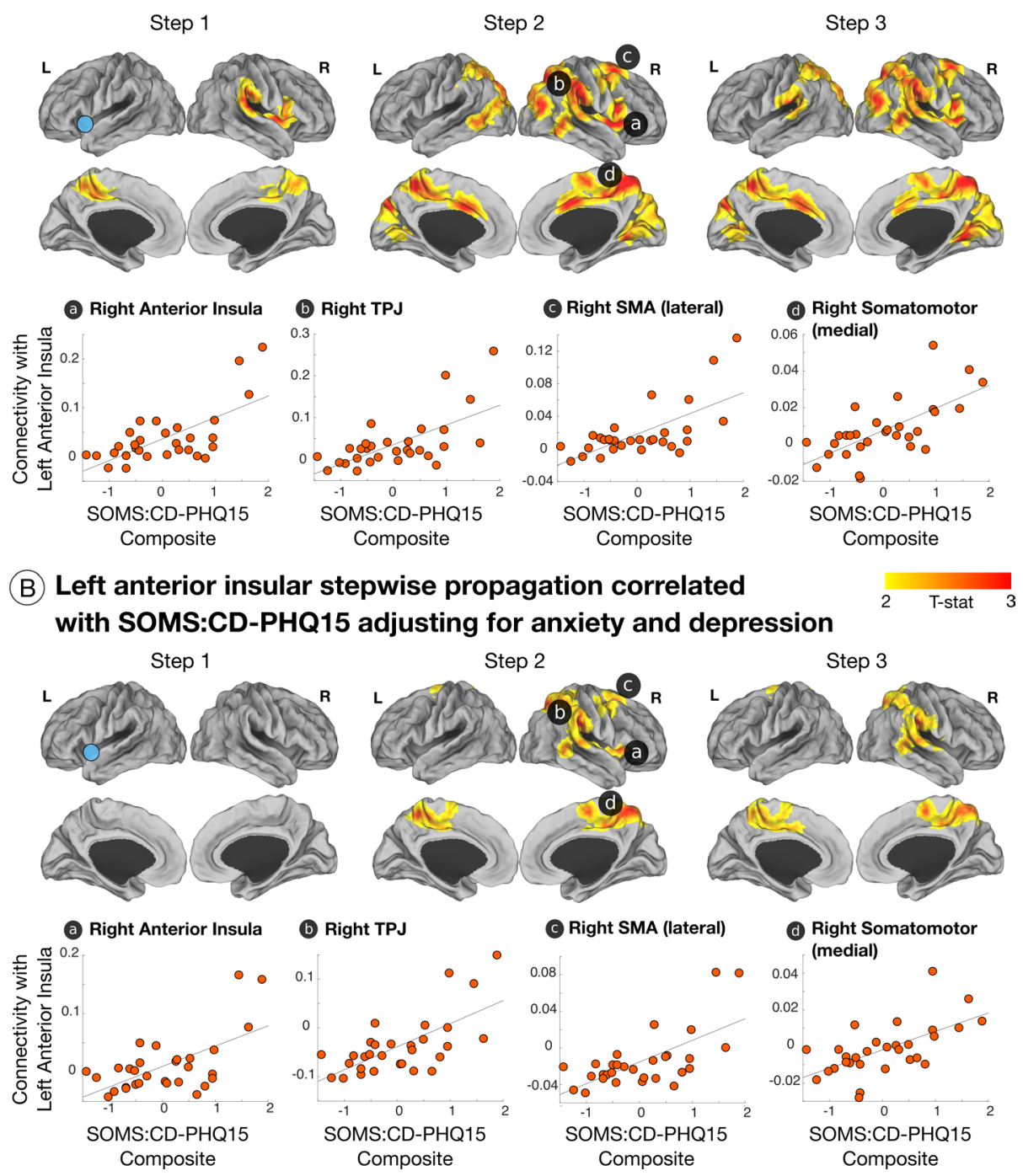

Figure 4 Insular stepwise functional connectivity correlated with symptom severity in patients with functional neurological disorder (FND). (A) First linkstep showed a positive correlation between patient-reported symptom severity and increased left anterior insula to right anterior insula, middle cingulate cortex, inferior frontal gyrus, temporoparietal junction (TPJ), bilateral precuneus and postcentral gyri link-step connectivity. For the second link-step, this correlation included the right anterior insula, inferior frontal gyrus, premotor, supplementary motor area (SMA), TPJ, bilateral dorsal anterior cingulate/ middle cingulate cortices, precentral/postcentral gyri and occipital areas. The third link-step showed similar results to the second link-step. Scatter plots display findings with a T statistic $>4$ in the second link-step. (B) Displays the correlations between patient-reported symptom severity and left anterior insula functional propagation profiles adjusting for anxiety and depression. PHQ15, Patient Health Questionnaire-15; SOMS:CD, Screening for Somatoform Symptoms-7 Conversion Disorder subscale.

Within-group symptom severity analyses

There were no statistically significant relationships between motor or amygdala SFC profiles and SOMS:CD-PHQ15 scores in patients with FND.

SOMS:CD-PHQ15 scores positively correlated with enhanced left anterior insula first link-step connectivity to the right anterior-middle insula, MCC, inferior frontal gyrus, TPJ, bilateral precuneus and postcentral gyri in patients with FND (figure 4). In the second link-step, FND symptom severity also positively correlated with increased left anterior insula link-step connectivity to the right anterior-middle insula, inferior frontal gyrus, premotor, SMA, TPJ, bilateral dorsal ACC/MCC, precentral/ postcentral gyri and occipital areas (figure 4). The third link-step showed similar findings to the second link-step. Of note, using Cook's distance there were no outliers.
In post hoc analyses adjusting separately for depression/ anxiety and SSRI/SNRI medication use, correlations between SOMS:CD-PHQ15 scores and left anterior insula enhanced second link-step connectivity to the right anterior insula, TPJ, bilateral precentral/postcentral gyri, and SMA remained statistically significant. Increased second and third link-step propagation to the dorsal ACC/MCC held adjusting for SSRI/SNRI medication use, but not for depression/anxiety. In post hoc analyses accounting for motor FND subtypes, FND symptom severity positively correlated with enhanced left anterior insula to right TPJ link-step connectivity across all subgroups; positive correlations between increased left to right anterior insula link-step connectivity and SOMS:CD-PHQ15 scores were most appreciable in those with functional weakness (online supplementary figure 4). 


\section{Left centromedial amygdalar propagation correlated with 6-month improvement}
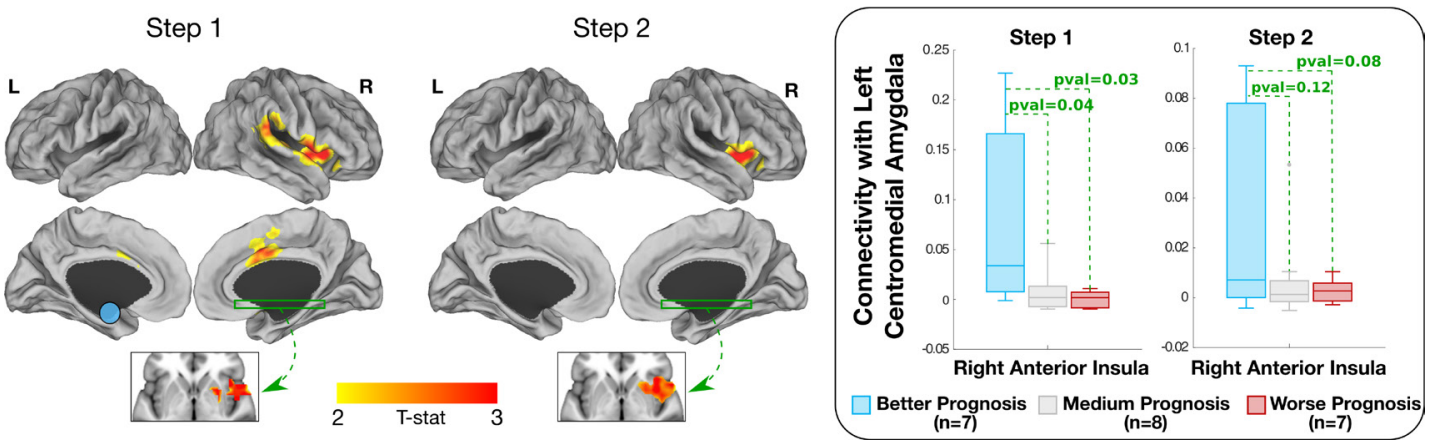

Figure 5 Pilot stepwise functional connectivity correlations with 6-month clinical improvement in patients with functional neurological disorder (FND). The first link-step showed a correlation between better 6 -month clinical outcomes and higher left centromedial amygdala connectivity to the right anterior insula, putamen, dorsal anterior cingulate cortex/middle cingulate cortex and temporoparietal junction (TPJ). For the second link-step, improvement positively correlated with left centromedial amygdala connectivity to the right anterior insula and putamen. In additional post hoc analyses, patients with FND were stratified in three groups: better prognosis $(n=7)$, medium prognosis $(n=8)$ and worse prognosis $(n=7)$. Boxplots show that the relationship between left centromedial amygdalar-right anterior insula first link-step stepwise functional connectivity and clinical improvement was driven by increased centromedial amygdala-anterior insula connectivity in the seven most improved individuals compared with the other subgroups.

Within-group naturalistic 6-month outcome pilot

Six-month improvement in SOMS:CD-PHQ15 scores positively correlated with left centromedial amygdalar first linkstep connectivity to the right anterior insula, dorsal ACC/ MCC, putamen and TPJ (figure 5). Baseline left centromedial amygdalar second link-step connectivity to the right anterior insula and putamen also predicted 6-month improvement in SOMS:CD-PHQ15 scores. No outliers were identified in this 22-subject cohort using Cook's distance. A post hoc stratified analysis ( 7 most improved, 7 least improved, 8 with medium-range outcomes) showed that the relationship between baseline left centromedial amygdala-right anterior insula first link-step SFC and clinical improvement was driven by increased centromedial amygdala-anterior insula connectivity in the seven most improved individuals compared with the other subgroups. In analyses adjusting separately for baseline depression/anxiety and antidepressant use, only associations between first link-step left centromedial amygdala-right anterior insula connectivity and 6-month improvement remained statistically significant.

Across the right M1-hand and left M1-foot ROIs, enhanced link-step connectivity to the left inferior temporal gyrus positively correlated with 6-month improvement in SOMS:CD-PHQ15 scores. Only the left M1-foot findings held adjusting for depression/anxiety and antidepressant use (see online supplementary figures 5 and 6). No other ROIs showed any statistically significant findings.

\section{DISCUSSION}

Consistent with hypotheses, patients with FND compared with controls showed enhanced functional propagation from primary motor areas and amygdalar nuclei to the multimodal integration network. Specifically, patients with FND exhibited increased link-step connectivity between motor regions and the bilateral posterior insula, MCC, TPJ and putamen. Increased link-step connectivity from the primary motor cortex to the bilateral MCC and right TPJ remained significant adjusting for grouplevel depression and anxiety scores. Beginning from the right laterobasal amygdala, the FND cohort also showed enhanced connectivity to the left anterior insula, bilateral parahippocampal/fusiform gyri, hippocampus, basal ganglia, PAG and hypothalamus; these findings, however, did not hold adjusting for depression and anxiety scores. An exploratory interconnector analysis demonstrated that link-steps to the bilateral anterior insula, dIPFC, putamen, PAG and hypothalamus were altered across motor and amygdalar pathways in patients with FND. In within-group analyses, patient-reported symptom severity positively correlated with enhanced link-step connectivity from the left anterior insula to the right anterior insula, TPJ, precentral and postcentral gyri and SMA. In a pilot analysis, increased baseline link-step connectivity from the left centromedial amygdala to the right anterior insula correlated with a more favourable 6-month prognosis. Both symptom severity and outcome-related within-group findings held adjusting for depression/anxiety and antidepressant use. Collectively, these findings support aberrant delivery of motor and limbic information to higher order multimodal integration areas in FND.

Patients with FND have been reported to exhibit an attentional bias for functional motor symptoms, ${ }^{42} 43$ loss of sensory attenuation $^{44}$ and impaired interoceptive accuracy. ${ }^{45}$ In addition, some individuals with paroxysmal functional symptoms demonstrate heightened somatic and autonomic arousal without the subjective experience of anxiety ('panic without panic'). ${ }^{46}$ Given the enhanced link-step connectivity from primary motor cortex to posterior insula and MCC observed in this study, along with heightened laterobasal amygdala to bilateral anterior insula SFC, these findings support that impaired interoceptive processing (posterior insula) and altered emotional and self-awareness (anterior insula) may be implicated in the pathophysiology of FND. ${ }^{47}$ Regarding loss of sensory attenuation previously identified in $\mathrm{FND}^{44}$ it is possible that enhanced primary motor cortex to posterior insula stepwise connectivity observed in our cohort relates to this phenomenon and warrants further inquiry. Furthermore, the insula and MCC are convergent zones for negative emotional processing, nociception and cognitive control, ${ }^{48}$ which may shed light on the neural mechanisms driving the multiplicity of sensorimotor, viscerosomatic, affective and cognitive symptoms frequently endorsed by FND populations. Our findings also suggest that enhanced functional propagation from primary motor cortex to posterior insula, and laterobasal amygdala to anterior insula, may be at the intersection of FND symptoms and negative affect, given that these relationships did not remain significant adjusting for depression 
and anxiety. Interoceptive awareness tasks, particularly probing interoception and emotion processing concurrently, are needed to clarify the observations of this study.

The finding of enhanced link-step connectivity from the right laterobasal amygdala to the PAG and hypothalamus in patients with FND delineates a potential candidate mechanistic pathway. The laterobasal amygdala receives thalamic sensory afferents, and through interneurons, connects to the centromedial nucleus of the amygdala (the output centre projecting to the PAG and hypothalamus). ${ }^{49}$ In healthy subjects, SFC propagation from the laterobasal amygdala showed strong interamygdalar connectivity at the first link-step (figure 1). By the second link-step in healthy controls, the laterobasal amygdala connected to the perigenual and dorsal ACC; particularly appreciable in the third link-step was downstream propagation to the PAG and hypothalamus. Given that physical events can precipitate functional neurological symptoms, ${ }^{50}$ and that some individuals with FND endorse sensory triggers, enhanced laterobasal (sensory) amygdalar link-step functional connectivity in patients with FND suggests a pathway for early sensory-amygdalar influence over the PAG. Notably, the PAG is implicated in pain modulation, homeostasis, autonomic responses and defensive behaviours (including tonic immobility and freezing). ${ }^{51}$ PAG hyperactivity has also been identified in patients with FND engaged in emotion processing. ${ }^{5}$ Increased laterobasal amygdala-PAG connectivity may also relate to sympathetic-parasympathetic imbalances characterised in FND cohorts, ${ }^{52}$ and enhanced amygdalarhypothalamic connectivity is consistent with reported links between threat vigilance and salivary cortisol levels in patients with FND. ${ }^{53}$

Primary motor areas exhibiting enhanced link-step connectivity to the posterior insula, SMA and putamen are consistent with published reports of heightened connectivity between motor execution and limbic-paralimbic areas in FND populations. $^{7811-1318}$ The exploratory interconnector analysis suggests that the bilateral anterior insula, dlPFC, putamen, PAG and hypothalamus may be common points of altered functional propagation across motor and amygdalar pathways. The dlPFC is implicated in executive control and top-down emotion regulation. Given that dlPFC-related functional alterations have been characterised in several FND studies, ${ }^{8} 1854$ non-invasive dlPFC modulation to modify motor-limbic activity trans-synaptically may be a therapeutic intervention warranting study.

In within-group analyses adjusting for depression/anxiety and antidepressant use, a positive correlation was observed between FND symptom severity and enhanced link-step connectivity from the left anterior insula to the right anterior insula, TPJ, SMA and sensorimotor areas. We have previously characterised reduced left anterior insular volume in patients with FND associated with symptom severity. ${ }^{28} 29$ Our functional propagation findings suggest that while the left anterior insula may be an important node in the pathophysiology of FND, a network perspective incorporating the impact of abnormal integration of information from the left anterior insula to the right anterior insula, TPJ and motor regions sheds additional light on brainsymptom severity relationships. In addition, tasked-based ${ }^{655}$ and resting-state neuroimaging abnormalities ${ }^{23-25}$ in the TPJ/inferior parietal lobule have been characterised in FND cohorts, and linked to impaired motor attention awareness and self-agency. Enhanced insular-TPJ link-step connectivity correlating with symptom severity in our study, and previously described taskbased $^{55}$ and functional connectivity alterations ${ }^{25}$ across insular and TPJ areas, suggest that these epicentres both play important roles in promoting altered awareness in individuals with FND.
To date, no published studies have identified functional MRI biomarkers of prognosis in patients with FND. In a pilot analysis, individual differences in link-step connectivity from the left centromedial (output) amygdala to the right anterior insula predicted 6-month improvement. Given that appreciating links between mood, psychosocial stressors and FND symptoms are core components of psychotherapy for FND, ${ }^{37}$ we speculate that relative increases in baseline connectivity between the centromedial (output) amygdala and anterior insula may be a marker of preserved emotional awareness that potentially aids treatment response. In addition, analyses showed a possible connection between baseline primary motor cortex-left inferior temporal gyrus link-step connectivity and outcome, which may relate to emotional-linguistic processing. Larger scale studies are needed to comprehensively elucidate biomarkers predicting clinical outcomes.

Limitations include modest sample size, psychiatric comorbidities, psychotropic medication use, phenotypic heterogeneity and sole reliance on patient-reported symptom severity measures. Given that patients with FND commonly have psychiatric comorbidities, future studies with psychiatric controls are needed to clarify our between-group findings, particularly given that some nodes in the neurobiology of FND (eg, insula, amygdala) may be at the intersection of affective and somatic symptoms. Studies characterising functional propagation profiles in mood/anxiety, trauma-related disorders and other functional medical syndromes are also needed to better contextualise the findings of this study. In addition, while individuals with FND frequently exhibit mixed symptoms and/or can develop new functional symptoms over the course of their illness, the use of a transdiagnostic research approach remains debated. ${ }^{3056}$ In this study, we showed that enhanced left anterior insula to right TPJ link-step connectivity correlated with symptom severity across motor subtypes, while links between FND symptom severity and increased left to right anterior insula connectivity were most appreciable in those with functional weakness. These findings suggest some common pathophysiologic elements across motor FNDs, as well as the potential for distinct neurobiological features. More research is needed to combine patient-reported scales with objective measures, as well as combining neuroimaging and questionnaire data with behavioural, neuroendocrine and autonomic measures. Furthermore, more research is needed to investigate the relationships between primary motor cortex functional propagation profiles and specific FND symptoms (eg, functional hand weakness vs functional gait). Serially collected longitudinal neuroimaging data are also needed to contextualise if the findings reported here are disease related or representative of compensatory mechanisms; additional research is needed to identify neurocircuit nodes that are essential for symptom generation, and those alterations that more closely relate to predisposing vulnerabilities and perpetuating factors. Furthermore, in addition to an important role for the multimodal integration network, altered self-referential processing and contextual appraisal warrant additional research in patients with FND.

In conclusion, we applied network graph-theory approaches to identify enhanced propagation from primary motor cortex and laterobasal amygdala to cortical-subcortical areas implicated in salience, interoception, stress responses and self/emotional awareness. Altered link-step functional connectivity from the left anterior insula to the right anterior insula and TPJ may relate to symptom severity, while centromedial amygdala-anterior insula interactions require more study as a possible outcome biomarker. These findings suggest potential candidate neurocircuit pathways in the pathophysiology of FND. 


\section{Author affiliations}

Neurology, Massachusetts General Hospital, Harvard Medical School, Boston, Massachusetts, USA

${ }^{2}$ Radiology, Athinoula A Martinos Center for Biomedical Imaging, Charlestown, Massachusetts, USA

${ }^{3}$ Neurotechnology Laboratory, Tecnalia Health, Derio, Bizkai, Spain

${ }^{4}$ Radiology and Nuclear Medicine, Gordon Center for Medical Imaging, Harvard Medical School, Boston, Massachusetts, USA

${ }^{5}$ Psychiatry, Beth Israel Deaconess Medical Center, Harvard Medical School, Boston, Massachusetts, USA

${ }^{6}$ Psychiatry and Neurology, Rhode Island Hospital, Brown Medical School, Providence, Rhode Island, USA

${ }^{7}$ Neurology and Psychiatry, Massachusetts General Hospital, Harvard Medical School, Charlestown, Massachusetts, USA

Contributors ID, LOT, JS and DLP designed the study. BW, RJ, JPO and DLP collected the data. ID performed all statistical analyses supervised by JS and DLP. All authors critically interpreted the results and discussed potential post hoc analyses. ID and DLP wrote the manuscript, and all authors critically reviewed, edited and approved the manuscript.

Funding DLP was funded by the National Institute of Mental Health Grant K23MH111983-02, Massachusetts General Hospital Physician-Scientist Development Award and the Sidney R Baer Jr Foundation. ID was supported by postdoctoral fellowship programme from the Basque Country Government. This study was also supported by the NIH shared instrument grant S10RR023043.

Competing interests $B C D$ is a consultant at Merck, Med Learning Group and Haymarket; royalties from Oxford University Press and Cambridge University Press; on the editorial board of Neuroimage: Clinical, Cortex, Hippocampus, Neurodegenerative Disease Management. WCL has served on the editorial boards of Epilepsia and Epilepsy, receives editor's royalties from the publication of Gates and Rowan's Nonepileptic Seizures, 3rd ed (Cambridge University Press, 2010) and 4th ed (2018); author's royalties for Taking Control of Your Seizures: Workbook and Therapist Guide (Oxford University Press, 2015); has received research support from the NIH (NINDS 5K23NS45902 (PI)), Rhode Island Hospital, the American Epilepsy Society (AES), the Epilepsy Foundation (EF), Brown University, the Siravo Foundation and the DoD (W81XWH-17-0169 (PI)); serves on the Epilepsy Foundation New England Professional Advisory Board; has received honoraria for the American Academy of Neurology Annual Meeting Annual Course; has served as a clinic development consultant at University of Colorado Denver, Cleveland Clinic, Spectrum Health and Emory University; and has provided medicolegal expert testimony. MSK is a one-time consultant at Forum Pharmaceuticals; editor for Schizophrenia Research. DLP received honoraria from Harvard Medical School, the American Academy of Neurology and the Movement Disorder Society.

Patient consent for publication Not required.

Ethics approval The Partners Human Research Committee approved the study. Provenance and peer review Not commissioned; externally peer reviewed.

\section{REFERENCES}

1 Bass C, Peveler R, House A. Somatoform disorders: severe psychiatric illnesses neglected by psychiatrists. Br J Psychiatry 2001;179:11-14.

2 Benbadis SR. The problem of psychogenic symptoms: is the psychiatric community in denial? Epilepsy Behav 2005;6:9-14.

3 Stone J, LaFrance WC, Levenson JL, et al. Issues for DSM-5: conversion disorder. Am J Psychiatry 2010;167:626-7.

4 Espay AJ, Aybek S, Carson A, et al. Current concepts in diagnosis and treatment of functional neurological disorders. JAMA Neurol 2018;75:1132-41.

5 Aybek S, Nicholson TR, O'Daly 0, et al. Emotion-motion interactions in conversion disorder: an fMRI study. PLoS One 2015;10:e0123273.

6 Aybek S, Nicholson TR, Zelaya F, et al. Neural correlates of recall of life events in conversion disorder. JAMA Psychiatry 2014;71:52-60.

7 Voon V, Brezing C, Gallea C, et al. Emotional stimuli and motor conversion disorder. Brain 2010;133:1526-36.

8 Morris LS, To B, Baek K, et al. Disrupted avoidance learning in functional neurological disorder: implications for harm avoidance theories. Neuroimage Clin 2017;16:286-94.

9 Hassa T, Sebastian A, Liepert J, et al. Symptom-specific amygdala hyperactivity modulates motor control network in conversion disorder. Neuroimage Clin 2017; 15:143-50.

10 Pick S, Goldstein LH, Perez DL, et al. Emotional processing in functional neurological disorder: a review, biopsychosocial model and research agenda. J Neurol Neurosurg Psychiatry 2019;90:704-11

11 Wegrzyk J, Kebets V, Richiardi J, et al. Identifying motor functional neurological disorder using resting-state functional connectivity. Neuroimage Clin 2018;17:163-8.
12 van der Kruijs SJM, Bodde NMG, Vaessen MJ, et al. Functional connectivity of dissociation in patients with psychogenic non-epileptic seizures. J Neurol Neurosurg Psychiatry 2012;83:239-47.

13 Li R, Liu K, Ma X, et al. Altered functional connectivity patterns of the insular subregions in psychogenic nonepileptic seizures. Brain Topogr 2015;28:636-45.

14 Voon V, Cavanna AE, Coburn K, et al. Functional neuroanatomy and neurophysiology of functional neurological disorders (conversion disorder). J Neuropsychiatry Clin Neurosci 2016:28:168-90.

15 Cojan Y, Waber L, Carruzzo A, et al. Motor inhibition in hysterical conversion paralysis. Neuroimage 2009;47:1026-37.

16 de Lange FP, Roelofs K, Toni I. Increased self-monitoring during imagined movements in conversion paralysis. Neuropsychologia 2007;45:2051-8.

17 Stone J, Zeman A, Simonotto E, et al. FMRI in patients with motor conversion symptoms and controls with simulated weakness. Psychosom Med 2007;69:961-9.

18 Voon V, Brezing C, Gallea C, et al. Aberrant supplementary motor complex and limbic activity during motor preparation in motor conversion disorder. Mov Disord 2011;26:2396-403.

19 Seignourel PJ, Miller K, Kellison I, et al. Abnormal affective startle modulation in individuals with psychogenic [corrected] movement disorder. Mov Disord 2007;22:1265-71.

20 Sepulcre J. Functional streams and cortical integration in the human brain. Neuroscientist 2014;20:499-508.

21 Sepulcre J, Sabuncu MR, Yeo TB, et al. Stepwise connectivity of the modal cortex reveals the multimodal organization of the human brain. J Neurosci 2012;32:10649-61.

22 Ortiz-Terán L, Diez I, Ortiz T, et al. Brain circuit-gene expression relationships and neuroplasticity of multisensory cortices in blind children. Proc Natl Acad Sci U SA 2017:114:6830-5.

23 Voon V, Gallea C, Hattori N, et al. The involuntary nature of conversion disorder. Neurology 2010;74:223-8.

24 Baek K, Doñamayor N, Morris LS, et al. Impaired awareness of motor intention in functional neurological disorder: implications for voluntary and functional movement. Psychol Med 2017;47:1624-36.

25 Maurer CW, LaFaver K, Ameli R, et al. Impaired self-agency in functional movement disorders: a resting-state fMRI study. Neurology 2016;87:564-70.

26 Sepulcre J, Sabuncu MR, Becker A, et al. In vivo characterization of the early states of the amyloid-beta network. Brain 2013;136:2239-52.

27 Carmona S, Hoekzema E, Castellanos FX, et al. Sensation-to-cognition cortical streams in attention-deficit/hyperactivity disorder. Hum Brain Mapp 2015:36:2544-57.

28 Perez DL, Williams B, Matin N, et al. Corticolimbic structural alterations linked to health status and trait anxiety in functional neurological disorder. J Neurol Neurosurg Psychiatry 2017;88:1052-9.

29 Perez DL, Matin N, Barsky A, et al. Cingulo-insular structural alterations associated with psychogenic symptoms, childhood abuse and PTSD in functional neurological disorders. J Neurol Neurosurg Psychiatry 2017;88:491-7.

30 Perez DL, Dworetzky BA, Dickerson BC, et al. An integrative neurocircuit perspective on psychogenic nonepileptic seizures and functional movement disorders: neural functional unawareness. Clin EEG Neurosci 2015:46:4-15.

31 Williams DT, Ford B, Fahn S. Phenomenology and psychopathology related to psychogenic movement disorders. Adv Neurol 1995;65:231-57.

32 Daum C, Hubschmid M, Aybek S. The value of 'positive' clinical signs for weakness, sensory and gait disorders in conversion disorder: a systematic and narrative review. J Neurol Neurosurg Psychiatry 2014;85:180-90.

33 LaFrance WC, Baker GA, Duncan R, et al. Minimum requirements for the diagnosis of psychogenic nonepileptic seizures: a staged approach: a report from the International League against epilepsy nonepileptic seizures Task Force. Epilepsia 2013:54:2005-18

34 Rief W, Hiller W. A new approach to the assessment of the treatment effects of somatoform disorders. Psychosomatics 2003;44:492-8.

35 Kroenke K, Spitzer RL, Williams JBW. The PHQ-15: validity of a new measure for evaluating the severity of somatic symptoms. Psychosom Med 2002;64:258-66.

36 Perez DL, Williams B, Matin N, et al. Anterior hippocampal grey matter predicts menta health outcome in functional neurological disorders: an exploratory pilot study. J Neurol Neurosurg Psychiatry 2018;89:1221-4.

37 Sharpe M, Walker J, Williams C, et al. Guided self-help for functional (psychogenic) symptoms: a randomized controlled efficacy trial. Neurology 2011;77:564-72.

38 Nielsen G, Stone J, Matthews A, et al. Physiotherapy for functional motor disorders: a consensus recommendation. J Neurol Neurosurg Psychiatry 2015;86:1113-9.

39 Buckner RL, Krienen FM, Castellanos A, et al. The organization of the human cerebellum estimated by intrinsic functional connectivity. J Neurophysiol 2011; 106:2322-45.

40 Amunts K, Kedo 0, Kindler M, et al. Cytoarchitectonic mapping of the human amygdala, hippocampal region and entorhinal cortex: intersubject variability and probability maps. Anat Embryol 2005;210:343-52.

41 Sepulcre J. An OP4 functional stream in the language-related neuroarchitecture. Cereb Cortex 2015;25:658-66.

42 Pareés I, Kassavetis P, Saifee TA, et al. 'jumping to conclusions' bias in functional movement disorders. J Neurol Neurosurg Psychiatry 2012;83:460-3. 
43 Pareés I, Saifee TA, Kassavetis P, et al. Believing is perceiving: mismatch between selfreport and actigraphy in psychogenic tremor. Brain 2012;135:117-23.

44 Pareés I, Brown $H$, Nuruki $A$, et al. Loss of sensory attenuation in patients with functional (psychogenic) movement disorders. Brain 2014;137:2916-21.

45 Ricciardi L, Demartini B, Crucianelli L, et al. Interoceptive awareness in patients with functional neurological symptoms. Biol Psychol 2016;113:68-74.

46 Goldstein LH, Mellers JDC. Ictal symptoms of anxiety, avoidance behaviour, and dissociation in patients with dissociative seizures. I Neurol Neurosurg Psychiatry 2006;77:616-21.

47 Craig ADB. How do you feel--now? The anterior insula and human awareness. Nat Rev Neurosci 2009;10:59-70.

48 Shackman AJ, Salomons TV, Slagter HA, et al. The integration of negative affect, pain and cognitive control in the cingulate cortex. Nat Rev Neurosci 2011;12:154-67.

49 LeDoux J. The amygdala. Curr Biol 2007;17:R868-74.

50 Pareés I, Kojovic M, Pires C, et al. Physical precipitating factors in functional movement disorders. J Neurol Sci 2014;338:174-7.
51 Roelofs K. Freeze for action: neurobiological mechanisms in animal and human freezing. Philos Trans R Soc Lond B Biol Sci 2017;372. doi:10.1098/rstb.2016.0206. [Epub ahead of print: 19 Apr 2017].

52 Maurer CW, Liu VD, LaFaver K, et al. Impaired resting vagal tone in patients with functional movement disorders. Parkinsonism Relat Disord 2016;30:18-22.

53 Bakvis P, Spinhoven P, Roelofs K. Basal cortisol is positively correlated to threat vigilance in patients with psychogenic nonepileptic seizures. Epilepsy Behav 2009:16:558-60.

54 de Lange FP, Toni I, Roelofs K. Altered connectivity between prefrontal and sensorimotor cortex in conversion paralysis. Neuropsychologia 2010:48:1782-8

55 Nahab FB, Kundu P, Maurer $C$, et al. Impaired sense of agency in functional movement disorders: an fMRI study. PLoS One 2017;12:e0172502.

56 Kanaan RAA, Duncan R, Goldstein LH, et al. Are psychogenic non-epileptic seizures just another symptom of conversion disorder? I Neurol Neurosurg Psychiatry 2017;88:425-9. 